\title{
New DAMA dark-matter window and energetic-neutrino searches
}

\author{
Dan Hooper, ${ }^{1}$ Frank Petriello, ${ }^{2}$ Kathryn M. Zurek, ${ }^{2}$ and Marc Kamionkowski ${ }^{3}$ \\ ${ }^{1}$ Theoretical Astrophysics, Fermi National Accelerator Laboratory, Batavia, Illinois 60510, USA \\ ${ }^{2}$ Physics Department, University of Wisconsin, Madison, Wisconsin 53706, USA \\ ${ }^{3}$ California Institute of Technology, Mail Code 130-33, Pasadena, California 91125, USA
}

(Received 5 September 2008; published 22 January 2009)

\begin{abstract}
Recently, the DAMA/LIBRA Collaboration has repeated and reinforced their claim to have detected an annual modulation in their signal rate, and have interpreted this observation as evidence for dark-matter particles at the $8.2 \sigma$ confidence level. Furthermore, it has also been noted that the effects of channeling may enable a weakly interacting massive particle (WIMP) that scatters elastically via spin-independent interactions from nuclei to produce the signal observed by DAMA/LIBRA without exceeding the limits placed by CDMS, XENON, CRESST, CoGeNT, and other direct-detection experiments. To accommodate this elastic-scattering explanation, however, the mass of the responsible dark-matter particle must be relatively light, $m_{\mathrm{DM}} \lesssim 10 \mathrm{GeV}$. Such dark-matter particles will become captured by and annihilate in the Sun at very high rates, leading to a potentially large flux of $\mathrm{GeV}$-scale neutrinos. We calculate the neutrino spectrum resulting from WIMP annihilations in the Sun and show that existing limits from SuperKamiokande can be used to close a significant portion of the DAMA region, especially if the dark-matter particles produce tau leptons or neutrinos in a sizable fraction of their annihilations. We also determine the spin-dependent WIMP-nuclei elastic-scattering parameter space consistent with DAMA. The constraints from Super-Kamiokande on the spin-dependent scenario are even more severe-they exclude any selfannihilating WIMP in the DAMA region that annihilates $1 \%$ of the time or more to any combination of neutrinos, tau leptons, or charm or bottom quarks.
\end{abstract}

DOI: 10.1103/PhysRevD.79.015010

PACS numbers: $95.35 .+\mathrm{d}$

\section{INTRODUCTION}

Recently, the DAMA Collaboration has provided further evidence for an annual modulation in the rate of nuclearrecoil events observed in their experiment [1]. Such a signal arises naturally from postulating weakly interacting massive particles (WIMPs) in the galactic halo that scatter from target nuclei in detectors. The annual modulation of the interaction rate results from the variation in the relative velocity of the Earth with respect to the galactic darkmatter halo as the Earth orbits the Sun. This changes the flux of dark-matter particles and their velocity distribution, with expected extrema occurring at June 2 and December 2. The DAMA experiment observes a maximum rate at low nuclear-recoil energies on May 24, plus or minus 8 days, and have accumulated enough data to put the significance of the observed modulation at approximately $8 \sigma$. Both the phase and amplitude of the signal are highly suggestive of WIMP interactions. The Collaboration has not been able to identify any other systematic effects capable of producing this signal, and have claimed that the annual modulation is a discovery of dark-matter. This claim has been controversial, partly because a number of other experiments appear to be in direct contradiction.

Several studies have attempted to reconcile the DAMA modulation signal with the null results of other directdetection experiments [2-7], assuming a spin-independent
WIMP-nucleus interaction. A feature common to the analyses of Refs. [2,4-6,8,9] is that an elastically scattering WIMP with a mass in the several-GeV range can satisfy the results of DAMA. (For an alternate explanation of the DAMA signal using an inelastic scattering channel, see Ref. [7].) The consistency of this region with the null results of CDMS [10], CRESST [11], CoGeNT [12], and XENON [13] depends on the details of how the DAMA recoil energy spectrum is fit. If the data is divided into the two bins 2-6 keVee and 6-14 keVee, where keVee is the electron-equivalent recoil energy, then DAMA and the null experiments can be simultaneously accommodated by 3 $8 \mathrm{GeV}$ WIMPs. If the DAMA modulation data is binned more finely, then the modulation in the $2-2.5 \mathrm{keVee}$ bin right at the DAMA threshold is difficult to reconcile with other direct-detection constraints. We refer the reader to the studies in Refs. [5,8,9] for further details. The allowed parameter region depends crucially on the occurrence of channeling in the NaI crystals of the DAMA apparatus, an effect noted in Ref. [14] and studied by the DAMA Collaboration [15].

In this paper, we consider the constraints to the new DAMA dark-matter parameter space that come from null searches for energetic neutrinos from the Sun [16]. If WIMPs scatter from nuclei in DAMA, then they will also scatter from nuclei in the Sun, be captured and thus annihilate therein, and thus produce energetic neutrinos that 
can be sought in experiments such as IceCube [17], AMANDA [18], Baksan [19], MACRO [20], ANTARES [21], and Super-Kamiokande [22]. The only caveat is that the energetic-neutrino spectra will depend on the WIMPannihiation products. Still, the range of neutrino spectra is bracketed, and so model-independent bounds can be sometimes obtained [23]. In particular, the Sun is composed primarily of protons (nuclei with spin), and so null neutrino searches should be especially constraining for an explanation of DAMA in terms of a WIMP with spin-dependent interactions, as shown in Ref. [24] for the old spindependent DAMA parameter space.

Here, we determine the regions of the mass-crosssection parameter space implied by the new DAMA results for a spin-dependent WIMP, and we determine parameter space (for both spin-dependent and spin-independent WIMPs) eliminated by null neutrino searches. In order to extend the analysis of Ref. [24] to the lower WIMP masses implied by channeling, we calculate the full neutrino energy spectra produced by decays of tau leptons and charm and bottom quarks, and we consider prompt annihilation to neutrinos. We also include the effects of neutrino mixing and the effects of WIMP evaporation from the Sun. The muon energy thresholds of ANTARES and IceCube are $10 \mathrm{GeV}$ or higher and are therefore not able to observe the neutrinos produced by a several-GeV WIMP. We therefore consider bounds arising from the Super-Kamiokande experiment, which can identify muons with energies as low as $1.6 \mathrm{GeV}$. The limits imposed by Baksan and MACRO are similar to those coming form Super-Kamiokande. We study the DAMA allowed region arising from both the twobin study of Ref. [5] and the parameter space arising from the full spectral analysis both with and without the 22.5 keVee bin $[8,9]$.

Our conclusions are that upper limits to the flux of energetic neutrinos from the Sun severely constrain the DAMA spin-independent parameter space if WIMPs annihilate directly to neutrinos or to tau leptons. The constraints from Super-Kamiokande on the spin-dependent scenario are even more severe; they exclude any spindependent WIMP in the DAMA region that annihilates $1 \%$ of the time or more to any combination of neutrinos, tau leptons, or charm or bottom quarks.

Our paper is organized as follows: We review in Sec. II the formalism of WIMP capture and annihilation in the Sun, including the effect of evaporation, which is important for dark matter near the $3-\mathrm{GeV}$ lower edge of the DAMA allowed region. In Sec. III, we discuss the detection of the neutrinos from WIMP annihilation using upward-going muons in the Super-Kamiokande detector and study the constraints imposed by this process on the DAMA allowed region. We also present in Sec. III the spin-dependent parameter space (including channeling) implied by DAMA. In Sec. IV, we comment on the energetic-neutrino constraint as applied to the case of light neutralino dark matter. Finally, we summarize our conclusions in Sec. V.

\section{WIMP CAPTURE AND ANNIHILATION IN THE SUN}

We briefly review here the basic formulae describing WIMP capture and annihilation in the Sun. A generic species of dark-matter particle present in the Solar System will scatter elastically with and become captured in the Sun at a rate given by [25]

$$
\begin{aligned}
C^{\odot \simeq} & 1.3 \times 10^{25} \mathrm{~s}^{-1}\left(\frac{\rho_{\mathrm{DM}}}{0.3 \mathrm{GeV} / \mathrm{cm}^{3}}\right)\left(\frac{270 \mathrm{~km} / \mathrm{s}}{\bar{v}}\right) \\
& \times\left(\frac{1 \mathrm{GeV}}{m_{\mathrm{DM}}}\right)\left[\left(\frac{\sigma_{\mathrm{H}}}{10^{-40} \mathrm{~cm}^{2}}\right) S\left(m_{\mathrm{DM}} / m_{\mathrm{H}}\right)\right. \\
& \left.+1.1\left(\frac{\sigma_{\mathrm{He}}}{16 \times 10^{-40} \mathrm{~cm}^{2}}\right) S\left(m_{\mathrm{DM}} / m_{\mathrm{He}}\right)\right],
\end{aligned}
$$

where $\rho_{\text {DM }}$ is the local dark-matter density, $\bar{v}$ is the local root-mean-square velocity of halo dark-matter particles, and $m_{\mathrm{DM}}$ is the dark-matter mass. $\sigma_{\mathrm{H}}$ and $\sigma_{\mathrm{He}}$ are the elastic-scattering cross sections of the WIMP with hydrogen and helium nuclei, respectively. The factor of 1.1 reflects the solar abundance of helium relative to hydrogen and well as dynamical factors and form factor suppression. The quantity $S$ is a kinetic suppression factor given by

$$
S(x)=\left[\frac{A(x)^{3 / 2}}{\left(1+A(x)^{3 / 2}\right)}\right]^{2 / 3},
$$

where

$$
A(x)=\frac{3 x}{2(x-1)^{2}}\left(\frac{\left\langle v_{\mathrm{esc}}\right\rangle}{\bar{v}}\right)^{2} .
$$

For WIMPs much heavier than hydrogen and helium nuclei, this suppression can be considerable. For WIMPs in the $1-10 \mathrm{GeV}$ range, however, $S \approx 0.9-1.0$.

If the capture rate and annihilation cross sections are sufficiently large, equilibrium will be reached between these processes. The differential equation governing the number of WIMPs in the Sun, denoted here as $N$, is

$$
\dot{N}=C^{\odot}-A^{\odot} N^{2}-E^{\odot} N,
$$

where $C^{\odot}$ is the capture rate, $A^{\odot}$ is the annihilation cross section times the relative WIMP velocity per unit volume, and $E^{\odot}$ is the inverse time for a WIMP to exit the Sun via evaporation. $A^{\odot}$ can be approximated by

$$
A^{\odot}=\frac{\langle\sigma v\rangle}{V_{\text {eff }}},
$$

where $V_{\text {eff }}$ is the effective volume of the core of the Sun determined roughly by matching the core temperature with the gravitational potential energy of a single WIMP at the core radius. This was found in Refs. $[26,27]$ to be 


$$
V_{\text {eff }}=5.7 \times 10^{30} \mathrm{~cm}^{3}\left(\frac{1 \mathrm{GeV}}{m_{\mathrm{DM}}}\right)^{3 / 2} .
$$

Neglecting evaporation for the moment, the present WIMP annihilation rate is given by

$$
\Gamma=\frac{1}{2} A^{\odot} N^{2}=\frac{1}{2} C^{\odot} \tanh ^{2}\left(t_{\odot} / \tau_{E}\right),
$$

where $t_{\odot} \simeq 4.5$ billion years is the age of the Solar System, and $\tau_{E}=\left(C^{\odot} A^{\odot}\right)^{-1 / 2}$ is the time required to reach equilibrium. The annihilation rate is maximized when it reaches equilibrium with the capture rate. This occurs when

$$
t_{\odot} / \tau_{E} \gg 1 \text {. }
$$

In the case in question, this condition will be met so long as

$$
\langle\sigma v\rangle \geq 3 \times 10^{-30} \mathrm{~cm}^{3} / \mathrm{s}\left(\frac{1 \mathrm{GeV}}{m_{\mathrm{DM}}}\right)^{1 / 2}\left(\frac{10^{-40} \mathrm{~cm}^{2}}{\sigma_{\mathrm{H}}}\right) .
$$

Once equilibrium is reached, the final annihilation rate (and corresponding neutrino flux and event rate) has no further dependence on the dark-matter particle's annihilation cross section.

For WIMPs with masses in the range being considered here, the process of WIMP evaporation from the Sun could also be potentially important. Ref. [28] found an approximate timescale for WIMP evaporation given by (see also Ref. [29])

$$
E^{\odot} \approx 10^{-\left((7 / 2)\left(m_{\mathrm{DM}} / \mathrm{GeV}\right)+4\right)} \mathrm{s}^{-1}\left(\frac{\sigma_{\mathrm{H}}}{5 \times 10^{-39} \mathrm{~cm}^{2}}\right) .
$$

The WIMP annihilation rate in the presence of evaporation is [29]

$$
\Gamma=\frac{1}{2} C^{\odot}\left[\frac{\tanh \left(\alpha t_{\odot} / \tau_{E}\right)}{\alpha+\frac{1}{2} E^{\odot} \tau_{E} \tanh \left(\alpha t_{\odot} / \tau_{E}\right)}\right]^{2},
$$

with $\alpha=\left\{1+\left(E^{\odot} \tau_{E} / 2\right)^{2}\right\}^{1 / 2}$. For the lightest range of WIMP masses in the DAMA region (3-4 GeV), the timescale for the process of WIMP evaporation can be comparable to the time required to reach capture-annihilation equilibrium, $E^{\odot} \tau_{E} \gtrsim 1$, and thus may potentially reduce the annihilation rate. In Fig. 1, we show the suppression of WIMP annihilation resulting from evaporation as a function of the WIMP's annihilation cross section, obtained from the ratio of Eqs. (7) and (11). For WIMPs heavier than $\sim 4 \mathrm{GeV}$, evaporation plays a negligible role. For lighter WIMPs, however, the annihilation rate and corresponding neutrino flux may be suppressed, depending on the magnitude of the annihilation cross section. In our calculations, we will use a dark-matter annihilation cross section of $\sigma v=3 \times 10^{-26} \mathrm{~cm}^{3} / \mathrm{s}$, which is generally expected for a thermal relic in the absence of resonant annihilation, coannihilations or strong $s$-wave suppression. If the annihilation cross section is smaller than this value, the effects of WIMP evaporation on the annihilation rate and corre-

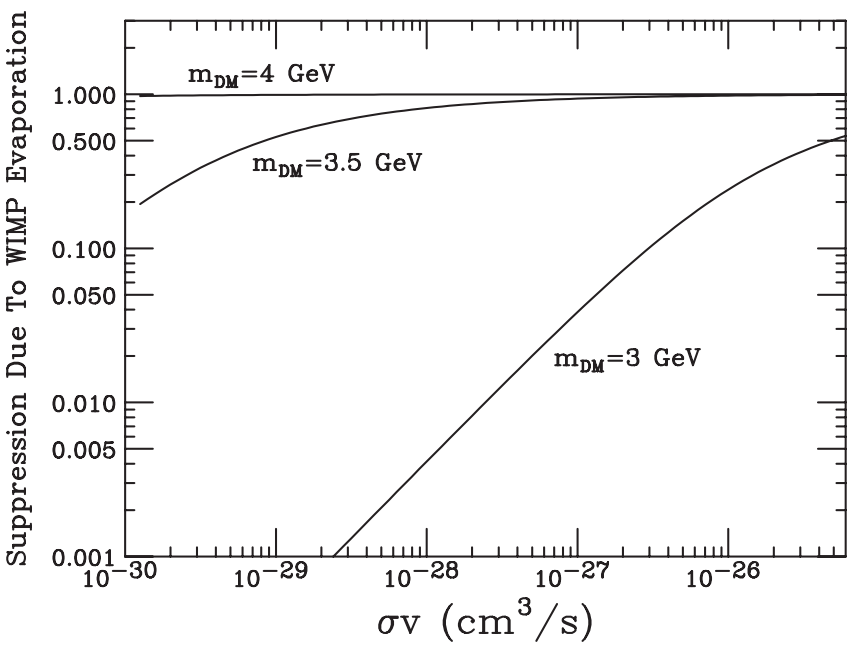

FIG. 1. The factor by which the WIMP annihilation rate in the Sun is suppressed as a result of WIMP evaporation. For each WIMP mass, we used a spin-independent elastic-scattering cross section near the middle of the DAMA region (see the upper frame of Fig. 3). For WIMPs heavier than $4 \mathrm{GeV}$, the effect of evaporation is negligible.

sponding neutrino flux may be more pronounced, as shown in Fig. 1.

\section{LIMITS FROM MUON PRODUCTION IN SUPER-KAMIOKANDE}

As the WIMPs annihilate, they can generate neutrinos through a wide range of channels. Annihilations to bottom quarks, charm quarks, and tau leptons each generate neutrinos in their subsequent decays. In some models, WIMPs can also annihilate directly to neutrino pairs. Once produced, neutrinos travel to the Earth where they can be detected. The spectra of muon neutrinos and antineutrinos at the Earth from WIMP annihilations in the Sun is given by

$$
\begin{gathered}
\frac{d N_{\nu_{\mu}}}{d E_{\nu_{\mu}}} \approx \frac{1}{3} \frac{C_{\odot} F_{\mathrm{Eq}}}{4 \pi D_{\mathrm{ES}}^{2}}\left(\frac{d N_{\nu_{e}}}{d E_{\nu_{e}}}+\frac{d N_{\nu_{\mu}}}{d E_{\nu_{\mu}}}+\frac{d N_{\nu_{\tau}}}{d E_{\nu_{\tau}}}\right)^{\mathrm{Inj}}, \\
\frac{d N_{\bar{\nu}_{\mu}}}{d E_{\bar{\nu}_{\mu}}} \approx \frac{1}{2} \frac{C_{\odot} F_{\mathrm{Eq}}}{4 \pi D_{\mathrm{ES}}^{2}}\left(\frac{d N_{\bar{\nu}_{\mu}}}{d E_{\bar{\nu}_{\mu}}}+\frac{d N_{\bar{\nu}_{\tau}}}{d E_{\bar{\nu}_{\tau}}}\right)^{\mathrm{Inj}},
\end{gathered}
$$

where $C_{\odot}$ is the WIMP capture rate in the Sun, $F_{\mathrm{Eq}}$ is the nonequilibrium suppression factor $(\approx 1$ for captureannihilation equilibrium), $D_{\mathrm{ES}}$ is the Earth-Sun distance, and the bracketed quantities are the neutrino and antineutrino spectra from the Sun per annihilating WIMP. Because of $\nu_{\mu}-\nu_{\tau}$ vacuum oscillations and Mikheyev, Smirnov, and Wolfenstein-enhanced $\nu_{e}$ oscillations [30] in the Sun, the muon neutrino flux is approximately given by the average of the $\nu_{\mu}, \nu_{e}$ and $\nu_{\tau}$ components, leading to the factor of $1 / 3$ in Eq. (12). The oscillations of antielectron neutrinos are Mikheyev, Smirnov, and Wolfenstein sup- 
pressed, however, leading to a flux of antimuon neutrinos, which is the average of the $\bar{\nu}_{\mu}$ and $\bar{\nu}_{\tau}$ components [31].

Muon neutrinos produce muons in charged-current interactions with nuclei inside of and around the detector volume of Super-Kamiokande. In the analysis of the SuperKamiokande Collaboration [22], upward-going muon tracks extending 7 meters or more within the inner detector were counted. Such events are produced through darkmatter annihilations in the Sun at a rate given by

$$
\begin{aligned}
R_{\text {events }} \approx & \iint \frac{1}{2} \frac{d N_{\nu_{\mu}}}{d E_{\nu_{\mu}}} \frac{d \sigma_{\nu}}{d y}\left(E_{\nu_{\mu}}, y\right) R_{\mu}\left(E_{\mu}\right) A_{\mathrm{Det} N_{A} d E_{\nu_{\mu}} d y} \\
& +\iint \frac{1}{2} \frac{d N_{\bar{\nu}_{\mu}}}{d E_{\bar{\nu}_{\mu}}} \frac{d \sigma_{\bar{\nu}}}{d y}\left(E_{\bar{\nu}_{\mu}}, y\right) R_{\mu}\left(E_{\mu}\right) A_{\operatorname{Det} N_{A} d E_{\bar{\nu}_{\mu}} d y .}
\end{aligned}
$$

Here, $\quad \sigma_{\nu}\left(E_{\nu_{\mu}}\right) \approx 8 \times 10^{-39} \mathrm{~cm}^{2} \times\left[E_{\nu} /(\mathrm{GeV})\right]$ and $\sigma_{\bar{\nu}}\left(E_{\bar{\nu}_{\mu}}\right) \approx 3 \times 10^{-39} \mathrm{~cm}^{2} \times\left[E_{\bar{\nu}} /(\mathrm{GeV})\right]$ are the neutrino-nucleon and antineutrino-nucleon chargedcurrent interaction cross sections, $(1-y)$ is the fraction of the neutrino's (or antineutrino's) energy, which goes into the muon, $A_{\text {Det }}$ is the effective area of the detector, $N_{A}$ is Avogadro's number, which for water is the number density of protons, and $R_{\mu}\left(E_{\mu}\right)$ is the range a muon travels before losing its energy. For the dimensions of the inner detector, we take $A_{\text {Det }}=900 \mathrm{~m}^{2}$ and a height of 36.2 meters, for a total target volume of $32500 \mathrm{~m}^{3}$, or 32.5 metric tons. To account for the 7-meter cut applied in the SuperKamiokande analysis, we substitute the physical muon range $\left(R_{\mu} \approx 5\right.$ meters $\left.\times E_{\mu} / \mathrm{GeV}\right)$, with zero if $R_{\mu}<$ 7 meters or otherwise with $R_{\mu}+(36.2-7)$ meters. This cutoff turns out to be irrelevant, however, since evaporation effects remove all light WIMPs with masses below $2 \mathrm{GeV}$ where it would be important. The factor of $1 / 2$ accounts for the Sun being below the Super-Kamiokande detector approximately $50 \%$ of the time.

For the injection spectrum of neutrinos produced in WIMP annihilations, we consider the following annihilation channels: $\nu \bar{\nu}, \tau^{+} \tau^{-}, c \bar{c}$, and $b \bar{b}$. We neglect annihilation products such as muon pairs, light mesons, etc. as they are expected to lose the vast majority of their energy before decaying and thus do not produce energetic neutrinos. For the case of annihilations to neutrino-antineutrino pairs, we have assumed that equal quantities of each flavor are produced. If instead all of the neutrinos and antineutrinos produced were of electron (muon or tau) flavor, the rate would be $27 \%$ smaller (14\% larger).

For annihilations to tau pairs, we include the semileptonic decays $\tau \rightarrow \mu \nu \nu, e \nu \nu$, as well as from the hadronic decays $\tau \rightarrow \pi \nu, K \nu, \pi \pi \nu$, and $\pi \pi \pi \nu$. For charm and bottom quarks, only semileptonic decays contribute. We model the neutrino energy spectra coming from these decays using updated versions of the formulae in Ref. [32] including tau and electron neutrinos. For the hadronic $\tau$ decays we use approximate formulae that reproduce reasonably well the results obtaining using HERWIG [33]. We neglect the possible production of quarkonia near the $b \bar{b}$ threshold and instead use the results for open-flavor semileptonic decay; as the neutrinos from $b$ decay do not impose strong constraints we believe this approximation is sufficient.

In Fig. 2, we plot the rate of events at SuperKamiokande predicted from annihilating WIMPs. In the upper frame, we consider spin-independent elastic scattering with nuclei, which in Eq. (1) corresponds to $\sigma_{\mathrm{H}}=$ $\sigma_{p, \mathrm{SI}}$ and $\sigma_{\mathrm{He}}=16 \sigma_{p, S I}$. In the lower frame, we consider spin-dependent scattering with $\sigma_{\mathrm{H}}=\sigma_{p, S D}$ and $\sigma_{\mathrm{He}}=0$ (we neglect the tiny contribution from spin-dependent scattering from ${ }^{3} \mathrm{He}$ ). For comparison, we also show as
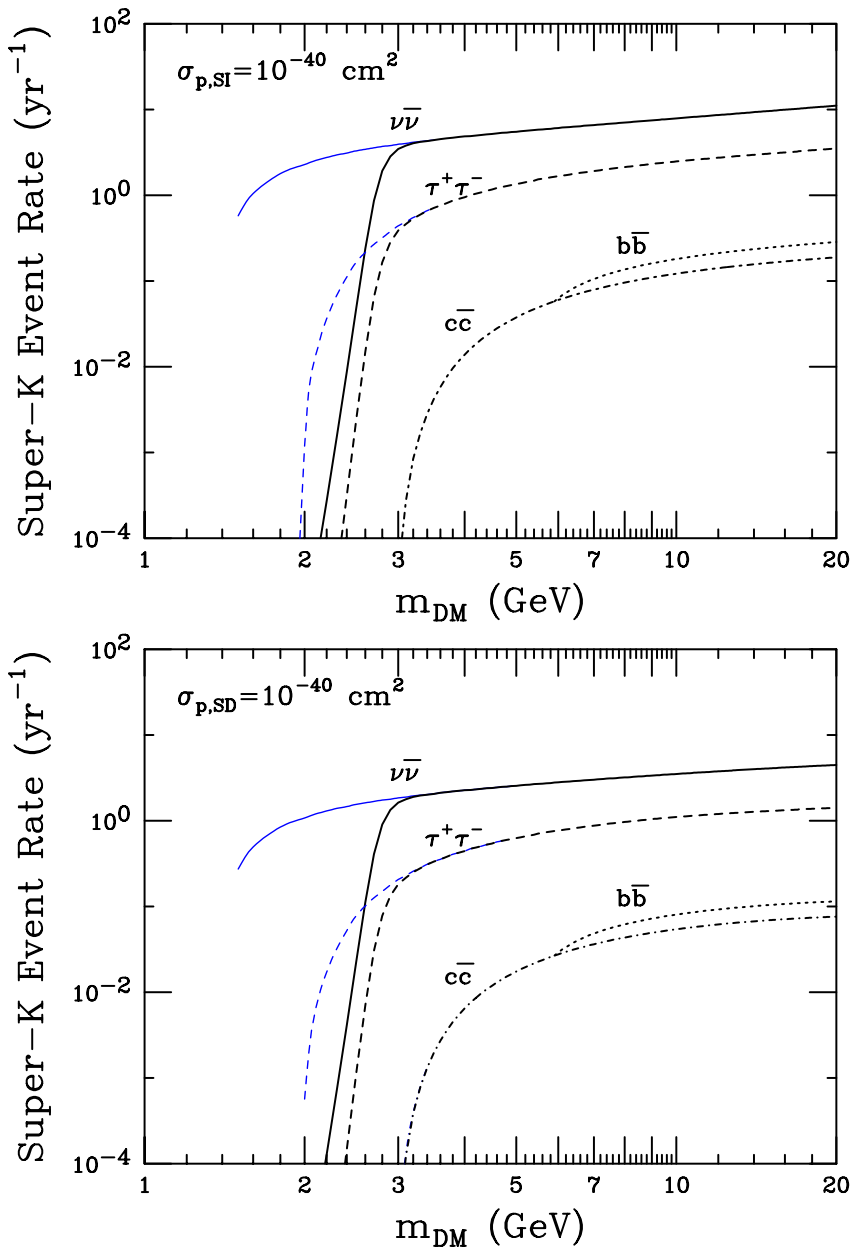

FIG. 2 (color online). The number of upward-going muon events per year in Super-Kamiokande from WIMPs annihilating in the Sun as a function of mass for an elastic-scattering cross section with protons of $10^{-40} \mathrm{~cm}^{2}$, assuming $100 \%$ annihilation to the indicated channel. The upper and lower frames correspond to spin-independent and spin-dependent couplings, respectively. In each frame, the thin (blue) lines extending to the left denote the results neglecting the effects of WIMP evaporation. See text for more details. 
thin (blue) lines in these frames the rate neglecting the effects of WIMP evaporation.

We can translate our predicted rate in SuperKamiokande to a limit on the WIMP elastic-scattering cross section as a function of mass and the dominant annihilation channel. According to the analysis of the Super-Kamiokande Collaboration [22], 170 events were observed within an angular window of radius $36^{\circ}$ centered around the Sun, over 1679.6 live days. Comparing this with the expected rate from atmospheric neutrinos of 185 events, this leads to a $2 \sigma$ upper limit on the contribution from WIMP annihilations of approximately $[170+$
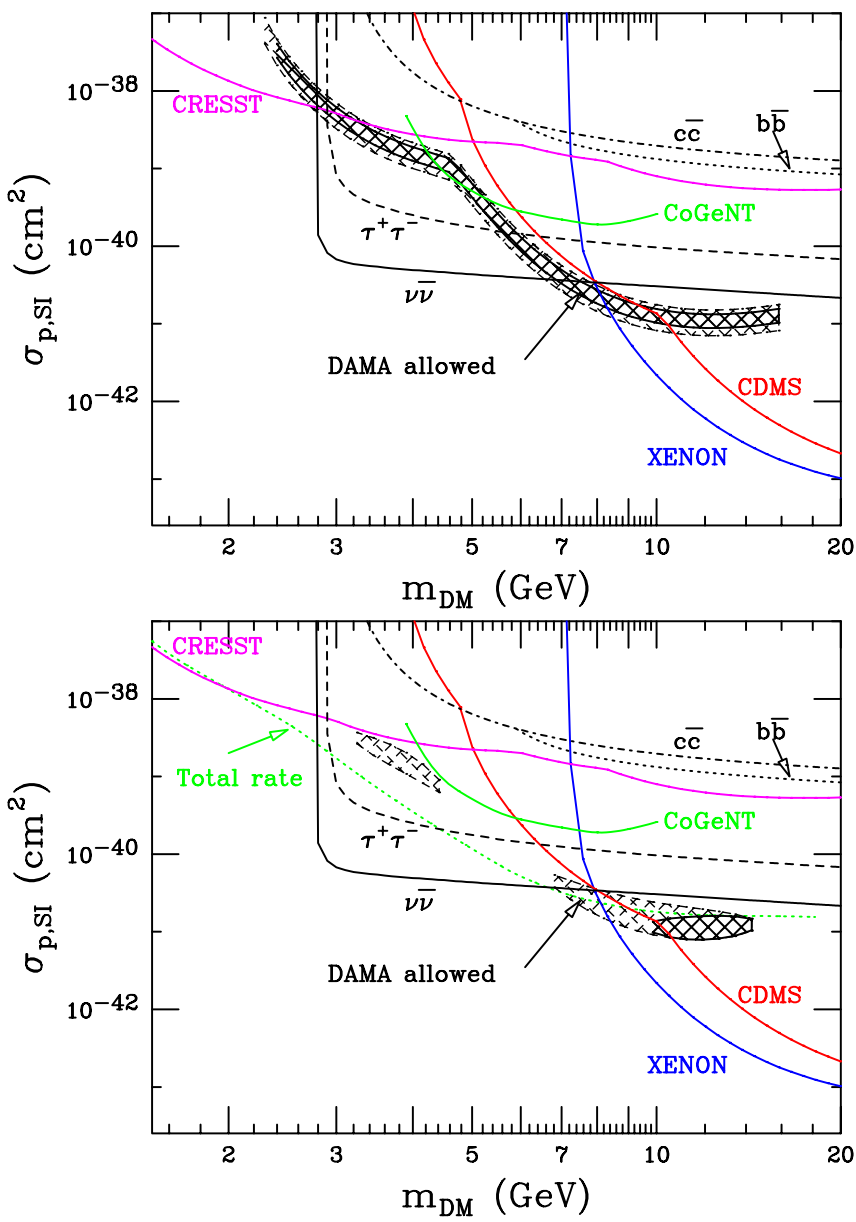

FIG. 3 (color online). The limit on a light WIMP's spinindependent elastic-scattering cross section with nuclei from Super-Kamiokande for various choices of dominant annihilation modes. The upper frame contains the DAMA allowed region as calculated in the two-bin analysis of Ref. [5], while the lower frame uses the full spectral analysis with (dark hatched region) and without (light hatched region) the 2-2.5 keVee bin. Also shown are the limits from the CDMS [10], CRESST [11], CoGeNT [12], and XENON [13] collaborations. The dotted green lines are the constraints derived by demanding that the predicted total rates predicted by a given WIMP candidate do not exceed those observed by DAMA at the $2 \sigma$ level in any energy bin.
$2 \sqrt{170}]-185 \approx 11$ events. We find a similar result is a somewhat smaller angular window is considered.

In Figs. 3 and 4, we apply this limit and plot our results in the $\left(\sigma, m_{\mathrm{DM}}\right)$ plane for the cases of spin-independent and spin-dependent scattering. We show both the two-bin analysis procedure of Ref. [5] and the full spectral analysis of the DAMA data both with and without the 2-2.5 keVee bin at threshold [9]. We include in these plots the constraint coming from demanding that the total rate observed by the DAMA Collaboration not exceed that predicted by a given WIMP mass and cross section. The form factors and couplings required for the calculation of the spin-dependent
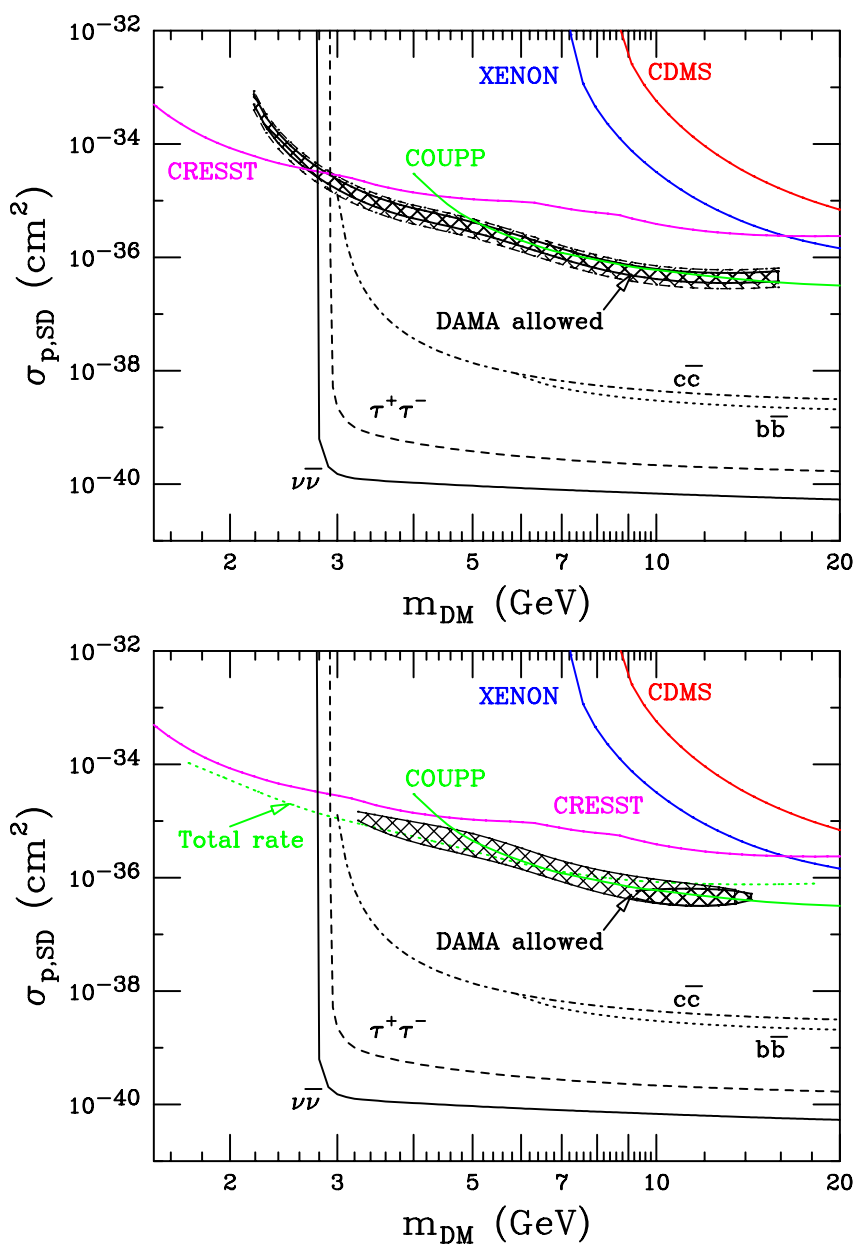

FIG. 4 (color online). The limit on a light WIMP's spindependent elastic-scattering cross section with nuclei from Super-Kamiokande for various choices of dominant annihilation modes. The upper frame contains the DAMA allowed region as calculated following the two-bin analysis of Ref. [5], while the lower frame uses the full spectral analysis with (dark hatched region) and without (light hatched region) the 2-2.5 keVee bin. Also shown are the limits from the CDMS [40], CRESST [11], XENON [41], and COUPP [42] collaborations. The dotted green lines are the constraints derived by demanding that the predicted total rates predicted by a given WIMP candidate do not exceed those observed by DAMA at the $2 \sigma$ level in any energy bin. 
DAMA allowed region were obtained from Refs. [34,35]. We find that if the WIMP annihilates to neutrinos or taus a significant fraction of the time, existing data from SuperKamiokande closes a large fraction of the DAMA window. In the case of spin-dependent scattering, WIMPs in the DAMA region that annihilate to neutrinos, tau leptons, or charm quarks or bottom quarks with any significant probability are ruled out by Super-Kamiokande. The only possible exception occurs near $m_{\mathrm{DM}} \approx 2.6-3.1 \mathrm{GeV}$, where evaporation enables the WIMP to evade detection by Super-Kamiokande.

\section{LIGHT NEUTRALINO DARK MATTER}

Within the context of the R-parity conserving minimal supersymmetric standard model (MSSM), a neutralino (if the lightest supersymmetric particle) in the mass range of the DAMA window would very likely be overproduced in the early Universe [36]. The main reason for this conclusion is that LEP constraints force the MSSM Higgs bosons, charginos and sfermions to be heavier than $\sim 100 \mathrm{GeV}$, in which case they are unable to efficiently mediate the process of neutralino annihilation or to participate in coannihilations. In supersymmetric models with extended Higgs sectors, however, this conclusion can be evaded. In the next-to-ninimal supersymmetric standard model, for example, it has been shown that very light neutralinos $(\sim 1-10 \mathrm{GeV})$ can be thermally produced in acceptable quantities [37] (see also Ref. [38]). This is made possible by the exchange of a relatively light pseudoscalar Higgs boson. Intriguingly, if the lightest Higgs scalar is very light and singletlike, light neutralinos are naturally predicted to possess an elastic-scattering cross section near or within the DAMA window.

Being Majorana fermions, neutralino annihilation to fermions is chirality suppressed [39], leading to $\sigma v \propto$ $m_{f}^{2} / m_{\chi^{0}}^{2}$. For neutralinos in the mass range under consideration here, annihilations proceed almost entirely to combinations of $b \bar{b}, \tau^{+} \tau^{-}$, and $c \bar{c}$. Above the bottom quark threshold, neutralino annihilations mediated via Higgs exchange produce bottom quarks approximately $3 m_{b}^{2} /\left(3 m_{b}^{2}+3 m_{c}^{2}+m_{\tau}^{2}\right) \sim 90 \%$ of the time. For neutralinos near the $b$ mass, however, the phase space for annihilations to $b \bar{b}$ is reduced and the fraction of annihilations producing tau leptons and charm quarks is enhanced. Below the $b \bar{b}$ threshold, at least $40 \%$ of neutralino annihilations proceed to $\tau^{+} \tau^{-}$(and a considerably larger fraction if the Higgs' couplings to down-type fermions are enhanced by $\tan \beta$ ).

Comparing this with the results shown in Figs. 3 and 4, we can place constraints on light neutralinos as the source of the DAMA signal. Considering the case of spinindependent scattering, neutralinos above the $b \bar{b}$ threshold $\left(4.2 \mathrm{GeV} \lesssim \mathrm{m}_{\chi^{0}} \lesssim 8 \mathrm{GeV}\right)$ annihilate mostly to bottom quarks and are not able to be constrained by SuperKamiokande beyond the corresponding limits from XENON, CDMS, and CoGeNT. Below the $b \bar{b}$ threshold ( $3 \mathrm{GeV} \lesssim m_{\chi^{0}} \lesssim 4.2 \mathrm{GeV}$ ), however, many or most neutralino annihilations produce tau pairs, allowing SuperKamiokande to exclude this range of neutralino masses. For spin-dependent scattering, the constraints from SuperKamiokande are even more severe. It is difficult to imagine any neutralino that could generate the observed DAMA signal through spin-dependent scattering without being in conflict with the constraints we have presented in this paper (the possible exception being a neutralino with a $\sim 3 \mathrm{GeV}$ mass, which could efficiently evaporate in the Sun, thus suppressing the annihilation rate).

\section{CONCLUSIONS}

In this paper, we have discussed the indirect detection of light WIMPs in the several-GeV range by their capture in the Sun and subsequent annihilation to neutrinos. Such dark-matter particles can potentially accommodate the annual modulation signal observed by DAMA/LIBRA and the null results of other direct-detection experiments, once the effects of channeling are accounted for. We consider the constraints that can be placed by the SuperKamiokande experiment on WIMPs in the DAMA window for the cases of prompt dark-matter annihilation to neutrinos, and secondary neutrinos produced by decays of tau leptons and charm and bottom quarks. The constraints found are significant, and impose stringent constraints on the DAMA allowed parameter space, particularly in the low mass end of the DAMA region. For the case of spinindependent elastic scattering of WIMPs with nuclei, darkmatter particles that annihilate to tau leptons or neutrinos a significant fraction of the time are excluded by SuperKamiokande measurements. For spin-dependent scattering, the constraints are more severe; any annihilation fraction to neutrinos, tau leptons, or charm or bottom quarks above the $10^{-2}$ level is ruled out.

\section{ACKNOWLEDGMENTS}

D. H. is supported by the Fermi Research Alliance, LLC under Contract No. DE-AC02-07CH11359 with the U.S. Department of Energy and by NASA Grant No. NNX08AH34G. M. K. is supported by DOE Grant No. DE-FG03-92-ER40701 and the Gordon and Betty Moore Foundation. F. P. and K. M.Z. are supported by DOE Grant No. DE-FG02-95ER40896. F. P. and K. M.Z. thank the Aspen Center for Physics, where part of this work was completed. 
[1] R. Bernabei et al. (DAMA Collaboration), Eur. Phys. J. C 56, 333 (2008).

[2] A. Bottino, F. Donato, N. Fornengo, and S. Scopel, Phys. Rev. D 77, 015002 (2008).

[3] R. Foot, Phys. Rev. D 78043529 (2008).

[4] J. L. Feng, J. Kumar, and L. E. Strigari, Phys. Lett. B 670, 37 (2008).

[5] F. Petriello and K. M. Zurek, J. High Energy Phys. 09 (2008) 047.

[6] A. Bottino, F. Donato, N. Fornengo, and S. Scopel, Phys. Rev. D 78, 083520 (2008).

[7] S. Chang, G. D. Kribs, D. Tucker-Smith, and N. Weiner, arXiv:0807.2250; D. R. Smith and N. Weiner, Phys. Rev. D 64, 043502 (2001); D. Tucker-Smith and N. Weiner, Phys. Rev. D 72, 063509 (2005).

[8] S. Chang, A. Pierce, and N. Weiner, arXiv:0808.0196.

[9] M. Fairbairn and T. Schwetz, arXiv:0808.0704.

[10] Z. Ahmed et al. (CDMS Collaboration), arXiv:0802.3530; D. S. Akerib et al. (CDMS Collaboration), Phys. Rev. Lett. 96, 011302 (2006); D.S. Akerib et al. (CDMS Collaboration), Phys. Rev. D 68, 082002 (2003).

[11] G. Angloher et al., Astropart. Phys. 18, 43 (2002).

[12] C. E. Aalseth et al., Phys. Rev. Lett. 101, 251301 (2008).

[13] J. Angle et al. (XENON Collaboration), Phys. Rev. Lett. 100, 021303 (2008).

[14] E. M. Drobyshevski, Mod. Phys. Lett. A 23, 3077 (2008).

[15] R. Bernabei et al., Eur. Phys. J. C 53, 205 (2008).

[16] J. Silk, K. Olive, and M. Srednicki, Phys. Rev. Lett. 55, 257 (1985); J. S. Hagelin, K. W. Ng, K. A. Olive, Phys. Lett. B 180, 375 (1986); M. Kamionkowski, Phys. Rev. D 44, 3021 (1991); L. Bergstrom, J. Edsjo, and P. Gondolo, Phys. Rev. D 58, 103519 (1998); V. D. Barger, F. Halzen, D. Hooper, and C. Kao, Phys. Rev. D 65, 075022 (2002); J. L. Feng, K. T. Matchev, and F. Wilczek, Phys. Rev. D 63, 045024 (2001).

[17] T. DeYoung (IceCube Collaboration), Int. J. Mod. Phys. A 20, 3160 (2005); J. Ahrens et al. (The IceCube Collaboration), Nucl. Phys. B, Proc. Suppl. 118, 371 (2003).

[18] M. Ackermann et al. (AMANDA Collaboration), Astropart. Phys. 24, 459 (2006).

[19] M. M. Boliev et al., Proceedings of the Interbnational Workshop on Aspects of Dark Matter in Astrophysics and Particle Physics, Heidelberg, Germany, 1996, edited by H. V. Klapdor-Kleingrothaus and Y. Ramachers (World Scientific, Singapore 1997).
[20] M. Ambrosio et al. (MACRO Collaboration), Phys. Rev. D 60, 082002 (1999).

[21] J. Hossl (ANTARES Collaboration), in Proceedings of the Identification of Dark Matter (IDM), Edinburgh, Scotland, 2004, edited by N. J.C. Spooner and V. Kudryavtsev (World Scientific, Hackensack, New Jersey, 2005); J. Brunner (ANTARES Collaboration), Nucl. Phys. B, Proc. Suppl. 145, 323 (2005).

[22] S. Desai et al. (Super-Kamiokande Collaboration), Phys. Rev. D 70, 083523 (2004); 70, 109901(E) (2004).

[23] M. Kamionkowski, K. Griest, G. Jungman, and B. Sadoulet, Phys. Rev. Lett. 74, 5174 (1995).

[24] P. Ullio, M. Kamionkowski, and P. Vogel, J. High Energy Phys. 07 (2001) 044.

[25] A. Gould, Astrophys. J. 388, 338 (1992).

[26] and D. Seckel, Nucl. Phys. B283, 681 (1987); B296, 1034 (E) (1988).

[27] A. Gould, Astrophys. J. 321, 571 (1987).

[28] A. Gould, Astrophys. J. 321, 560 (1987).

[29] K. Griest and D. Seckel, Nucl. Phys. B283, 681 (1987);

[30] L. Wolfenstein, Phys. Rev. D 17, 2369 (1978); S. P. Mikheyev and A. Y. Smirnov, Sov. J. Nucl. Phys. 42, 913 (1986).

[31] M. Cirelli, N. Fornengo, T. Montaruli, I. Sokalski, A. Strumia, and F. Vissani, Nucl. Phys. B727, 99 (2005); B790, 338(E) (2008).

[32] G. Jungman and M. Kamionkowski, Phys. Rev. D 51, 328 (1995).

[33] D. Grellscheid and P. Richardson, arXiv:0710.1951.

[34] M. T. Ressell and D.J. Dean, Phys. Rev. C 56, 535 (1997).

[35] D. R. Tovey, R. J. Gaitskell, P. Gondolo, Y. Ramachers, and L. Roszkowski, Phys. Lett. B 488, 17 (2000).

[36] D. Hooper and T. Plehn, Phys. Lett. B 562, 18 (2003); A. Bottino, F. Donato, N. Fornengo, and S. Scopel, Phys. Rev. D 68, 043506 (2003).

[37] J.F. Gunion, D. Hooper, and B. McElrath, Phys. Rev. D 73, 015011 (2006).

[38] F. Ferrer, L. M. Krauss, and S. Profumo, Phys. Rev. D 74, 115007 (2006).

[39] H. Goldberg, Phys. Rev. Lett. 50, 1419 (1983).

[40] D. S. Akerib et al. (CDMS Collaboration), Phys. Rev. D 73, 011102 (2006).

[41] J. Angle et al., Phys. Rev. Lett. 101, 091301 (2008).

[42] E. Behnke et al. (COUPP Collaboration), Science 319, 933 (2008). 